\title{
Annular-Shaped Emission from Gallium Nitride
}

\section{Nanotube Lasers}

Changyi Li $i^{1}$, Sheng Liu ${ }^{2,3}$, Antonio Hurtado ${ }^{4}$, Jeremy B. Wright ${ }^{1,2}$, Huiwen X ${ }^{1}$, Ting Shan Luk ${ }^{2,3}$,

Jeffrey J. Figiel ${ }^{2}$, Igal Brener ${ }^{2,3}$, Steven. R. J. Brueck ${ }^{1}$, George T. Wang ${ }^{2 *}$

${ }^{1}$ Center for High Technology Materials, University of New Mexico, 1313 Goddard St. SE, Albuquerque, NM, 87106, USA.

${ }^{2}$ Sandia National Laboratories, Albuquerque, New Mexico 87185, USA.

${ }^{3}$ Center for Integrated Nanotechnology, Sandia National Laboratories, Albuquerque, New Mexico 87185, USA.

${ }^{4}$ Institute of Photonics, Physics Department, University of Strathclyde, Wolfson Centre, 106 Rottenrow East, Glasgow, G4 0NW, United Kingdom.

KEYWORDS: nanotube, laser, GaN, annular emission

ABSTRACT Annular-shaped lasing emission is demonstrated from gallium nitride nanotubes fabricated using a two-step top-down technique. By optically pumping, we observe a clear threshold of $1055 \mathrm{~kW} / \mathrm{cm}^{2}$, a narrow spectral linewidth of $0.19 \mathrm{~nm}$, and guided emission from the 
nanotubes. Lasing is also demonstrated in a liquid environment, with an approximate doubling in threshold observed. The nanotube lasers could be of interest for optical nanofluidic applications or applications benefitting from a hollow beam shape. More generally, the results indicate that cross-sectional shape control can be employed to manipulate the properties of nanolasers.
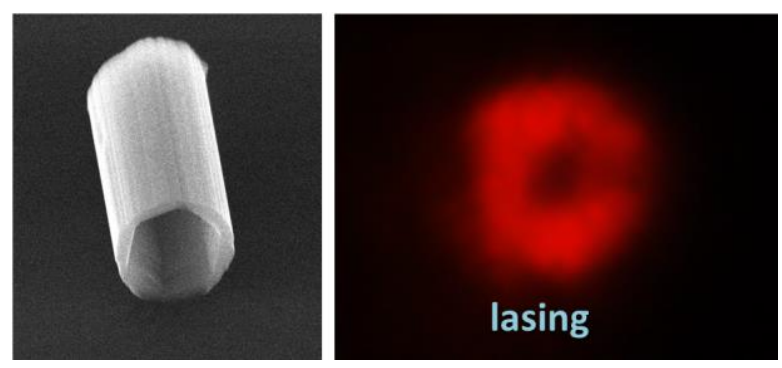

\section{Table of Contents Figure}

Semiconductor nanowire lasers have been explored as potential compact and low power requirement coherent light sources for a wide range of applications such as on-chip optical interconnects, sensors, and nanoscale probes ${ }^{1-5}$. In order to maximize the potential of nanowire lasers, a greater understanding and control over their properties, including mode control, polarization control, wavelength tuning, and beam shaping, is necessary. Recently, single mode emission from GaN nanowire lasers was demonstrated via several routes, including size (diameter and length) control $^{6}$, coupled nanowires ${ }^{7-9}$, distributed feedback ${ }^{10}$, and placement onto a metal substrate, which induces a mode-selective loss ${ }^{11}$. Wavelength tuning of GaN nanowire lasers was demonstrated using $\mathrm{GaN} / \mathrm{InGaN}$ nanowire photonic crystal arrays ${ }^{12,13}$, hydrostatic pressure $^{14}$, and bandgap graded nanowires ${ }^{15,16}$. and The polarization properties and control of 
polarization in GaN nanowire lasers were also studied via increasing optical excitation ${ }^{17}$ and a metal-induced mode-selection mechanism ${ }^{18}$.

The ability to control the cross-section of nanowire lasers could also provide novel routes to broaden their applications and manipulate their optical properties. Research on nanofluidic systems have opened a new window for many applications, such as analytical separation and determination of biomolecules ${ }^{19}$. Inorganic nanotubes can be used as ultracompact channels for nanofluidic devices ${ }^{20}$. Up to now, the study of the impact of nanowire geometry on lasing properties has been relatively unexplored, limited in part by difficulties in precisely and arbitrarily controlling cross-sectional shape using bottom-up nanowire growth methods. In this letter, we demonstrate optically pumped lasing from hollow-cross-section, GaN nanotubes fabricated by a two-step top-down technique.

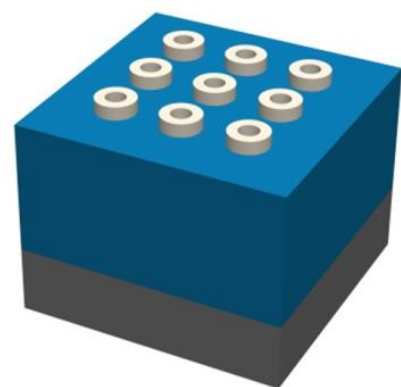

(a)

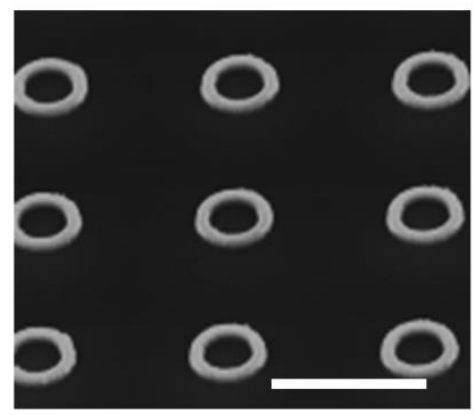

(d)

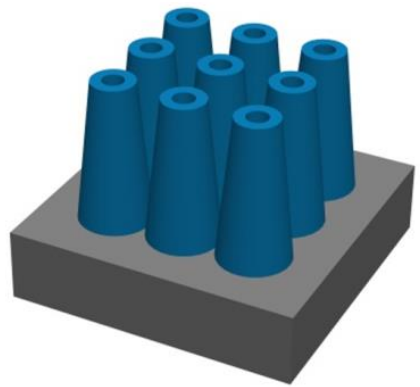

(b)

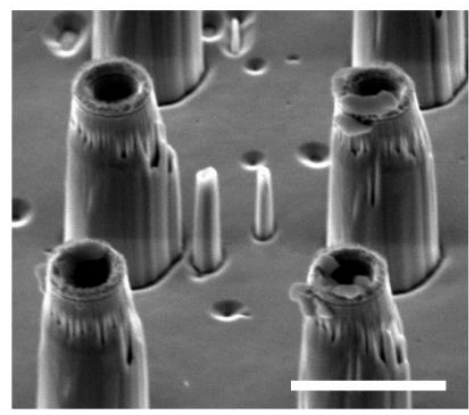

(e)

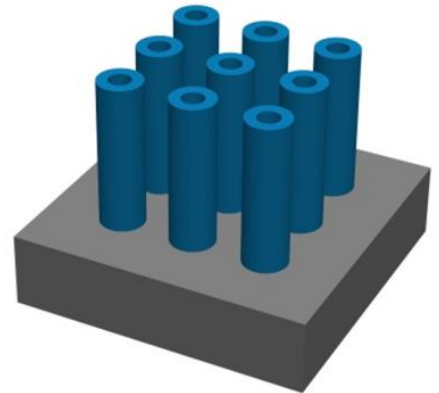

(c)

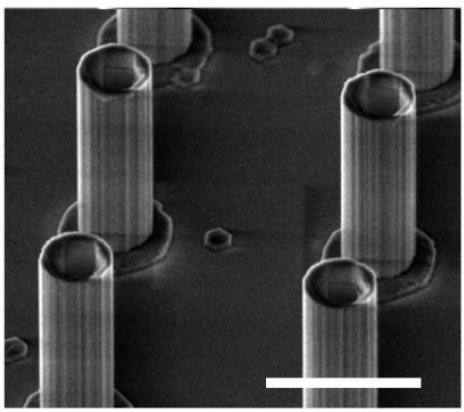

(f)

Figure 1. Sketches and corresponding SEM images of the fabrication process of GaN nanotube lasers. (a \& d) The deposition of the etch mask composed of nickel rings on top of the GaN 
layer. (b \& e) The ICP dry etch process. (c \& f) The selective KOH-based wet etch process. Scale bars: $3 \mu \mathrm{m}$.

The GaN nanotubes were fabricated by a top-down two-step etch process shown in Figure 1, which was initially developed for fabricating GaN nanowires ${ }^{6,21,22}$ and has been adapted here to create $\mathrm{GaN}$ nanotubes. First, a $4.3 \mu \mathrm{m}$ thick planar c-plane (0001) GaN film is grown on top of a 2-inch c-plane sapphire substrate by metal organic chemical vapor deposition. After the growth, a positive e-beam resist (495 polymethyl methacrylate (PMMA) C6 from MicroChem) is spincoated on top of the GaN film and electron beam lithography was employed to define annular shaped patterns. Next, a $350 \mathrm{~nm}$ thick, electron-beam-evaporated nickel film is deposited on the sample as an etch mask, leaving Ni rings on the GaN sample after PMMA lift-off, as seen in Figs. 1(a) and 1(d). A chlorine-based inductively coupled plasma (ICP) dry etch process is then used to transfer the nickel pattern into the GaN film, forming nanotubes as shown in Figs. 1(b) and 1(e). However, the sidewalls of the nanotubes after the dry etch process are rough and tapered, which results in significant optical scattering and thus losses. In order to improve the sidewall smoothness, create straight-sidewalls, and remove the dry etch damaged sidewall material, a $\mathrm{KOH}$ based (AZ400K developer) crystallographic selective wet etch process is subsequently applied, which results in c-axis oriented GaN nanotubes with smooth and straight sidewalls, as shown in Figs. 1(c) and 1(f) ${ }^{6,21}$. The fabricated nanotubes have outer diameters of $\sim 1.3 \mu \mathrm{m}$, lengths of $\sim 4.3 \mu \mathrm{m}$, and shell thicknesses of $\sim 75-150 \mathrm{~nm}$ (The shell thickness varies due to the hexagonal inner cross-section of the GaN nanotube lasers). In contrast to GaN nanotubes previously synthesized using an epitaxial casting method and selective area growth method ${ }^{23}$, the tubes fabricated via this top-down method are open at both ends, facilitating potential nanofluidic applications. 
The GaN nanotube lasers are characterized experimentally using a 2-arm custom microphotoluminescence ( $\mu$-PL) system ${ }^{17,18}$. The GaN nanotube lasers are optically excited by a 266 $\mathrm{nm}$, frequency quadrupled $\mathrm{Nd}$ :YAG microchip laser with a pulse width of $400 \mathrm{ps}$ and a repetition rate of $10 \mathrm{kHz}$. In order to vary the pump power density, a variable neutral density (ND) filter is placed after the pump laser. After passing through the ND filter, the pump laser beam is focused to a spot with a beam waist diameter of $\sim 3.5 \mu \mathrm{m}$ by means of a $50 \times$ infinity corrected objective. Due to the $5 \mu \mathrm{m}$ spacing between adjacent nanotubes, this spot size allows for individually pumping the GaN nanotubes. This objective is also used to collect the end-facet emission from the as-fabricated $\mathrm{GaN}$ nanotube lasers or the scattered emission from $\mathrm{GaN}$ nanotube lasers lying on the substrate. Images of the end-facet emission are captured by the CCD camera in the pump arm. The spectra of the GaN nanotube lasers are measured by a 2400 groove $/ \mathrm{mm}$ holographic grating spectrometer. For measurement of the polarization of the end-facet emission, GaN nanotubes were transferred to a cleaved edge of a silicon substrate ${ }^{10,17}$. Another polarizer is placed behind to measure the polarization of the GaN nanotube's emission.

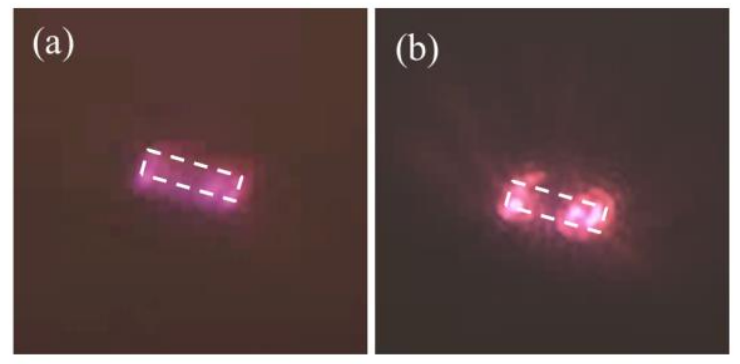

Figure 2. CCD images of a single $\mathrm{GaN}$ nanotube laser pumped (a) below and (b) above threshold. Dashed rectangles indicate the location of the GaN nanotube. Relatively uniform emission across the entire $\mathrm{GaN}$ nanotube is observed, which indicates spontaneous emission from the GaN nanotube. Stronger intensities of the emission at both ends of the nanotube than in the middle are observed when the GaN nanotube is excited above threshold, indicating a highly 
guided Fabry-Perot mode. The interference fringes in (b) also verify the spatial coherent emission from the GaN nanotube.

Different spatial intensity distributions of the emission from a GaN nanotube lying flat on a sapphire substrate are observed when it is optically pumped below and above lasing threshold (Figure 2). When the excitation level is below the lasing threshold, the intensity of the emission at both ends of the nanotube is slightly stronger than at the center, resulting in nearly uniform emission, because only partial spontaneous emission is coupled into the guided mode of the tubular waveguide. The absence of interference fringes also indicates incoherent emission. However, when the GaN nanotube is excited above a certain threshold level, the intensities of the emission at both ends of the nanotube become much stronger than in the middle due to the strongly guided mode (Figure 2(b)). This intensity distribution also implies that the GaN nanotube lases in a Fabry-Perot mode rather than a whispering gallery mode as reported for GaN nanorings grown by selective area growth using molecular beam epitaxy ${ }^{24}$. Interference fringes are also observed, indicating spatially coherent emission. 


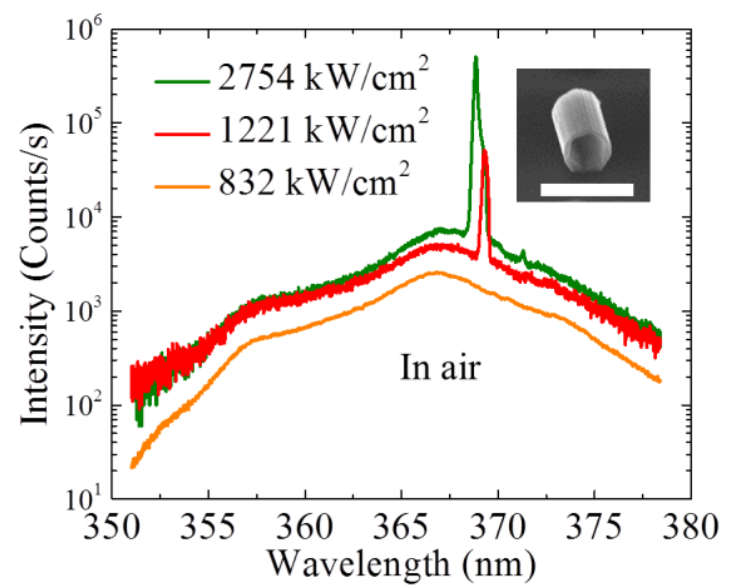

(a)

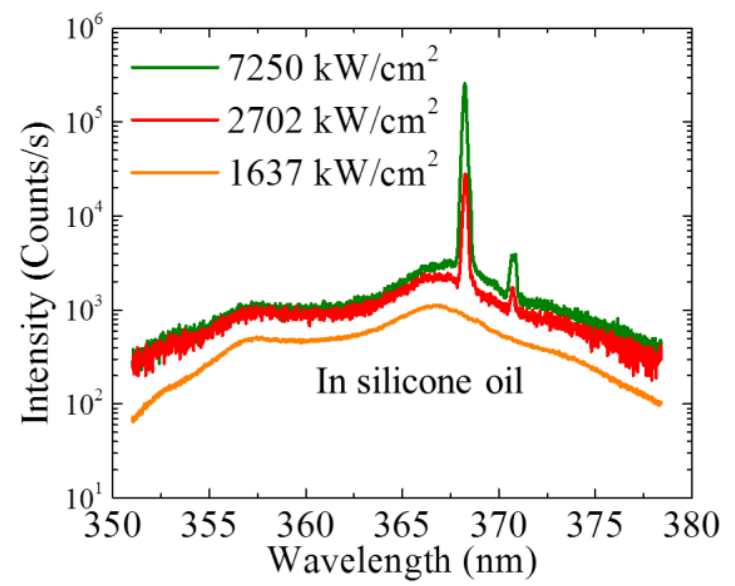

(b)

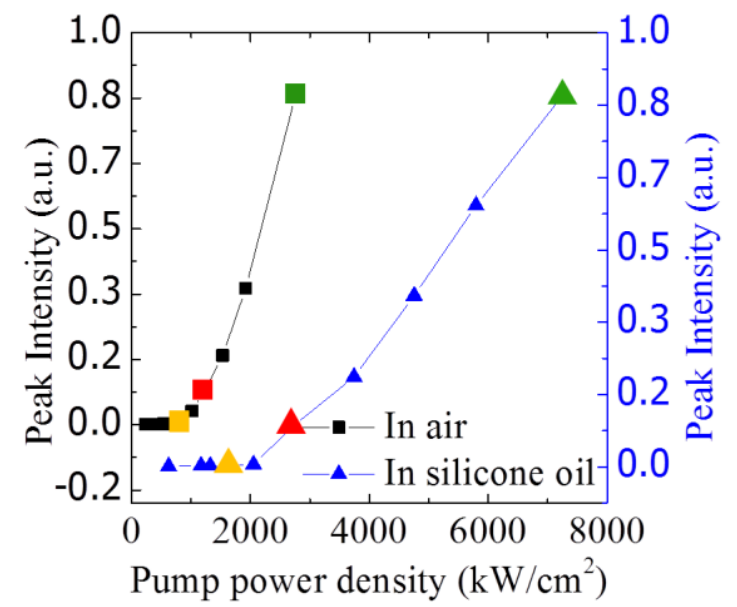

(c)

Figure 3. Spectra of the emission from a GaN nanotube laser (a) in air and (b) in silicone oil at different optical pump power density. (c) The peak intensity curve of the GaN nanotube laser 
versus pump power density (L-L curve). The colors of the boxes correspond to the colored spectra in (a) and (b). The L-L curve indicates lasing thresholds of $1055 \mathrm{~kW} / \mathrm{cm}^{2}$ in air and $2208 \mathrm{~kW} / \mathrm{cm}^{2}$ in silicone oil. Inset: SEM image of the measured GaN nanotube laser. The scale bar represents $2 \mu \mathrm{m}$.

Emission spectra of a $\mathrm{GaN}$ nanotube laser lying on a $\mathrm{Si}_{3} \mathrm{~N}_{4} / \mathrm{Si}$ substrate are shown in Figure 3 (a). At the lower pump power density $\left(832 \mathrm{~kW} / \mathrm{cm}^{2}\right)$, a broad band spectrum with a full width half maximum (FWHM) of $\sim 7 \mathrm{~nm}$ centered at $366 \mathrm{~nm}$ is observed, suggesting spontaneous emission from the GaN nanotube. At a pump level of $2754 \mathrm{~kW} / \mathrm{cm}^{2}$, the FWHM of the spectrum shows a dramatic reduction to $\sim 0.2 \mathrm{~nm}$. The relatively large linewidth results from multi-mode lasing from the GaN nanotube. Since the outer diameter of the GaN nanotube is much larger than half of the optical wavelength in GaN, the nanotube laser shows multi-transverse mode lasing behavior, with a mode spacing smaller than the resolution of the spectrometer, as seen in Figure 3(a). A blue-shifting of the peak wavelength with increasing pump power is also observed, which may be the result of mode-hopping ${ }^{17}$.

The intensity of the dominant lasing peak is plotted versus pump power density as shown in the light-in-light-out (L-L) curves in Figure 3(b) to more accurately estimate the lasing threshold. At lower pump power density, spontaneous emission dominates; therefore the peak intensity increases linearly as the pump power density increases. When the GaN nanotube is excited over a pump power density of approximately $1055 \mathrm{~kW} / \mathrm{cm}^{2}$, the peak intensity increases with a much larger slope as the pump power density increases. This implies that stimulated emission dominates and that the nanotube is excited above lasing threshold.

In order to demonstrate potential use for nanofluidic applications, the same hollow GaN nanotube laser was subsequently immersed in silicone oil ( refractive index $\sim 1.5$ ) and optically 
characterized. Lasing is observed from the GaN nanotube laser (Figure 3(c)) when the optical pump power density reaches the lasing threshold $\left(2208 \mathrm{~kW} / \mathrm{cm}^{2}\right)$ (Figure $3(\mathrm{~b})$ ), an approximate doubling from the threshold in air. The lasing threshold increase is due to the increase of ambient refractive index, which results in increase of mirror loss and decrease of confinement factor.

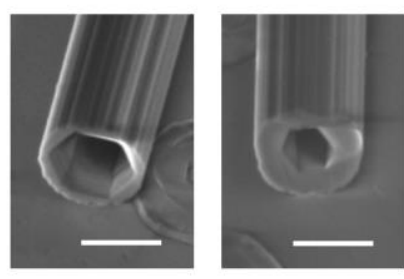

(a)

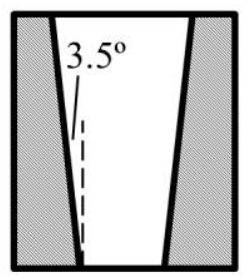

(b)

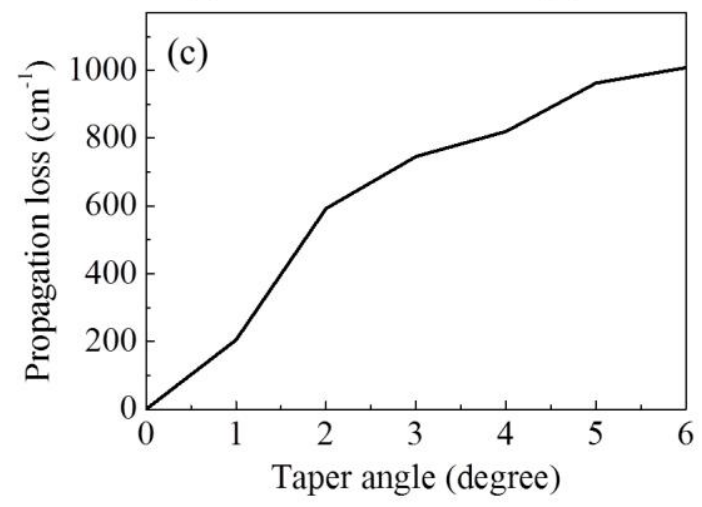

Figure 4. (a) SEM images of the top (left) and bottom (right) end-facets of a GaN nanotube. Scale bars: $1 \mu \mathrm{m}$. (b) Schematic diagram of the tapered inner sidewall of the GaN nanotube laser. The taper angle is calculated by measuring the inner diameters of the two end-facets. (b) Simulated propagation loss as a function of taper angle. A taper angle of 3.5 degree causes a propagation loss of about $800 \mathrm{~cm}^{-1}$.

We note that the measured lasing threshold of the nanotubes is significantly higher than reported for $c$-axis GaN nanowires fabricated by this two-step top-down approach ${ }^{6,11}$. In order to understand the threshold increase, an eigenmode solver (Lumerical Inc.) was used to simulate the confinement factor and the propagation loss of the transverse modes. The results show that, for a 
$\mathrm{GaN}$ nanotube laser, the electro-magnetic fields of the transverse modes are well-confined in the shell region, leading to only small electro-magnetic fields in the hollow region. Therefore, only a small difference of $\sim 2-3 \%$ in confinement factors of the transverse modes of a $1.3 \mu \mathrm{m} \mathrm{GaN}$ nanowire and a GaN nanotube are observed. The reflectivity of modes in a nanotube laser structure was also simulated by a finite different time domain (FDTD) simulation (Lumerical) and compared with a solid nanowire structure. The FDTD simulation shows that there is no significant difference in reflectivity that will significantly increase the lasing threshold compared to nanowires. However, we note that the GaN nanotubes show inward-tapered inner sidewalls from top to bottom likely due to reduced dry and wet etch efficiency inside of the tubes toward their bottoms. Both end-facets of 5 different nanotubes were imaged by SEM (Figure 4(a)) with an average $\sim 3.5$ degree of tapering measured. We performed simulations which show that the tapered inner sidewall results in propagation loss due to the mismatch between the transverse modes supported by cross-sections with different dimensions. The propagation loss of the fundamental mode is plotted as a function of taper angle of the inner sidewall in Figure 4 (c), showing that a 3.5 degree taper can cause an additional propagation loss of about $800 \mathrm{~cm}^{-1}$.

In order to analyze the modal properties of the nanotube lasers, the second collection arm of the $\mu$-PL was used to measure the polarization of the emission from a nanotube hanging off the edge of a silicon substrate ${ }^{17,18}$. Figure 5 (a) shows the spectra of the GaN nanotube at two orthogonal polarization angles $\left(80^{\circ}\right.$ and $\left.170^{\circ}\right)$. The intensities of the lasing peaks are plotted versus polarization angle in Figure 5 (b) and (c), respectively. Two different cross polarization suppression ratios are observed corresponding to two lasing peak groups, $\sim 5.4: 1$ for the shorter wavelength group and $\sim 1.4: 1$ for the longer wavelength group. The different cross polarization suppression ratios indicate that multi-transverse mode lasing occurs in the GaN nanotube laser. 
Figure 6 (a) shows the CCD image of end-facet emission from the GaN nanotube laser - notably, an annular shape emission is observed, although the GaN nanotube laser shows multi-transverse mode lasing.

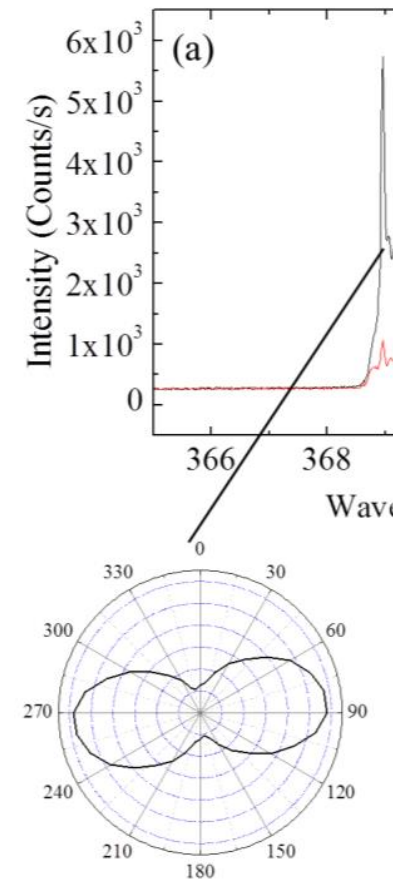

(b)

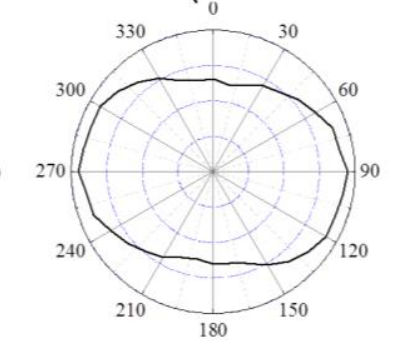

(c)

Figure 5. (a) Spectra of the end-facet emission from a "hanging" GaN nanotube at two polarization angles $\theta$. (b) Peak intensity of the shorter wavelength peaks versus polarization angle. (c) Peak intensity of the longer wavelength peaks versus polarization angle. Two different cross polarization suppression ratios are observed corresponding to two lasing peak groups. The different cross polarization suppression ratios indicate that multi-transverse mode lasing occurs in the GaN nanotube laser.

Transverse modes of the GaN nanotube laser were calculated by an eigenmode solver (Lumerical Inc). The simulation shows that over 100 non-degenerate transverse modes are supported by the GaN nanotube laser. The transverse modes are well-confined in the GaN shell region rather than the hollow air region. In order to understand the origin of the annular shaped 
lasing emission, FDTD simulations were utilized to calculate the far-field pattern resulting from the combination of all the transverse modes. The wavelength dependent refractive index for GaN used in the simulation was measured by spectral ellipsometry ${ }^{10}$. Two electric dipoles and two magnetic dipoles are randomly placed in the $\mathrm{GaN}$ nanotube to excite the optical modes supported by the GaN nanotube. Perfectly matched layer boundary condition is applied five wavelengths away from the GaN nanotube to minimize the interference of the boundary. The simulated farfield pattern is shown in Figure 6 (b). The simulated far-field emission from the GaN nanotube is annular shaped, consistent with the experimental result, with a divergence angle of $\sim 15^{\circ}$.

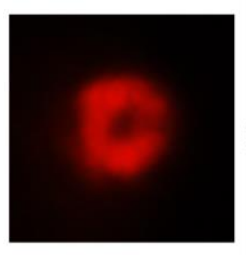

(a)

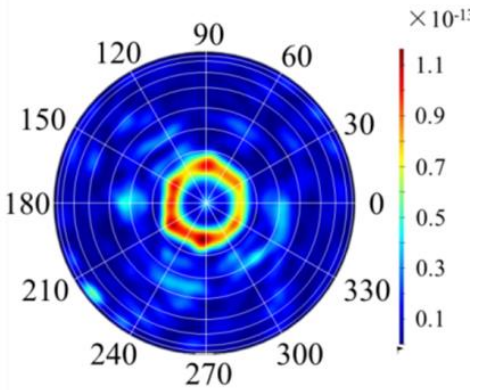

(b)

Figure 6. (a) CCD image of the end-facet emission of the GaN nanotube laser. (b) Simulation results of the far-field intensity of the GaN nanotube laser in spherical coordinate system.

In conclusion, $\mathrm{GaN}$ nanotubes with controlled dimensions and hollow ends are fabricated by two-step top-down etch process. The nanotubes lase under optical pumping at room temperature in both ambient and liquid environments, and show an observed annular end-facet emission pattern, opening the door for novel optical nano- and micro-fluidic applications. This donutshaped emission emerges from the annular cross-section of the nanotube and offers the possibilities for nano- and micro-photonic applications benefitting from a hollow beam shape such as on-chip atom trapping ${ }^{25}$ and stimulated emission depletion spectroscopy ${ }^{26}$. More 
generally, the results indicate that cross-sectional shape control of nanolasers can be used to tailor their optical properties.

AUTHOR INFORMATION

\section{Corresponding Author}

*E-mail: gtwang@,sanida.gov

\section{ACKNOWLEDGMENT}

This work is supported by Sandia's Solid-State-Lighting Science Energy Frontier Research

Center, funded by the U. S. Department of Energy, Office of Science, Office of Basic Energy

Sciences. The e-beam patterning was funded by Sandia's Laboratory Directed Research and

Development program. Dr. Antonio Hurtado acknowledges support by the European Commission under the Programme FP7 Marie Curie International Outgoing Fellowships (IOF) Grant PIOF-GA-2010-273822. This work was performed, in part, at the Center for Integrated Nanotechnologies, a U.S. Department of Energy, Office of Basic Energy Sciences user facility.

Sandia National Laboratories is a multi-program laboratory managed and operated by Sandia Corporation, a wholly owned subsidiary of Lockheed Martin Corporation, for the U.S. Department of Energy's National Nuclear Security Administration under contract DE-AC0494AL85000.

\section{REFERENCES}

(1) Arafin, S.; Liu, X.; Mi, Z. Review of Recent Progress of III-Nitride Nanowire Lasers. $J$. Nanophotonics 2013, 7, 074599.

(2) Johnson, J. C.; Choi, H.-J.; Knutsen, K. P.; Schaller, R. D.; Yang, P.; Saykally, R. J. Single Gallium Nitride Nanowire Lasers. Nat. Mater. 2002, 1, 106-110. 
(3) Cui, Y.; Wei, Q.; Park, H.; Lieber, C. M. Nanowire Nanosensors for Highly Sensitive and Selective Detection of Biological and Chemical Species. Science 2001, 293, 1289-1292.

(4) Yan, R.; Gargas, D.; Yang, P. Nanowire Photonics. Nat. Photonics 2009, 3, 569-576.

(5) Ma, Y.; Guo, X.; Wu, X.; Dai, L.; Tong, L. Semiconductor Nanowire Lasers. Adv. Opt. Photonics 2013, 5, 216-273.

(6) Li, Q.; Wright, J. B.; Chow, W. W.; Luk, T. S.; Brener, I.; Lester, L. F.; Wang, G. T. Single-Mode GaN Nanowire Lasers. Opt. Express 2012, 20, 17873-17879.

(7) Xu, H.; Wright, J. B.; Luk, T.-S.; Figiel, J. J.; Cross, K.; Lester, L. F.; Balakrishnan, G.; Wang, G. T.; Brener, I.; Li, Q. Single-Mode Lasing of GaN Nanowire-Pairs. Appl. Phys. Lett. 2012, 101, 113106.

(8) Xiao, Y.; Meng, C.; Wu, X.; Tong, L. Single Mode Lasing in Coupled Nanowires. Appl. Phys. Lett. 2011, 99, 023109.

(9) Gao, H.; Fu, A.; Andrews, S. C.; Yang, P. Cleaved-Coupled Nanowire Lasers. Proc. Natl. Acad. Sci. U. S. A. 2013, 110, 865-869.

(10) Wright, J. B.; Campione, S.; Liu, S.; Martinez, J. a.; Xu, H.; Luk, T. S.; Li, Q.; Wang, G. T.; Swartzentruber, B. S.; Lester, L. F.; Brener, I. Distributed Feedback Gallium Nitride Nanowire Lasers. Appl. Phys. Lett. 2014, 104, 041107.

(11) Xu, H.; Wright, J. B.; Hurtado, A.; Li, Q.; Luk, T.-S.; Figiel, J. J.; Cross, K.; Balakrishnan, G.; Lester, L. F.; Brener, I.; Wang, G. T. Gold Substrate-Induced SingleMode Lasing of GaN Nanowires. Appl. Phys. Lett. 2012, 101, 221114.

(12) Wright, J. B.; Liu, S.; Wang, G. T.; Li, Q.; Benz, A.; Koleske, D. D.; Lu, P.; Xu, H.; Lester, L.; Luk, T. S.; Brener, I.; Subramania, G. Multi-Colour Nanowire Photonic Crystal Laser Pixels. Sci. Rep. 2013, 3, 2982.

(13) Kishino, K.; Nagashima, K.; Yamano, K. Monolithic Integration of InGaN-Based Nanocolumn Light-Emitting Diodes with Different Emission Colors. Appl. Phys. 2013, 6, $2-4$.

(14) Liu, S.; Li, C.; Figiel, J. J.; Brueck, S. R. J.; Brener, I.; Wang, G. T. Continuous and Dynamic Spectral Tuning of Single Nanowire Lasers with Subnanometer Resolution Using Hydrostatic Pressure. Nanoscale 2015, 7, 9581-9588.

(15) Yang, Z.; Wang, D.; Meng, C.; Wu, Z.; Wang, Y.; Dai, L.; Liu, X.; Liu, X.; Yang, Q. Broadly Defining Lasing Wavelengths in Single Bandgap-Graded Semiconductor Nanowires. Nano Lett. 2014, 14, 3153-3159. 
(16) Li, J.; Meng, C.; Liu, Y.; Wu, X.; Lu, Y.; Ye, Y.; Dai, L.; Tong, L.; Liu, X.; Yang, Q. Wavelength Tunable CdSe Nanowire Lasers Based on the Absorption-EmissionAbsorption Process. Adv. Mater. 2013, 25, 833-837, 832.

(17) Hurtado, A.; Xu, H.; Wright, J. B.; Liu, S.; Li, Q.; Wang, G. T.; Luk, T. S.; Figiel, J. J.; Cross, K.; Balakrishnan, G.; Lester, L. F.; Brener, I. Polarization Switching in GaN Nanowire Lasers. Appl. Phys. Lett. 2013, 103, 251107.

(18) Xu, H.; Hurtado, A.; Wright, J. B.; Li, C.; Liu, S.; Figiel, J.; Luk, T.; Brueck, S. R. J.; Brener, I.; Balakrishnan, G.; Li, Q.; Wang, G. T. Polarization Control in GaN Nanowire Lasers. Opt. Express 2014, 22, 19198-19203.

(19) Cao, H.; Tegenfeldt, J. O.; Austin, R. H.; Chou, S. Y. Gradient Nanostructures for Interfacing Microfluidics and Nanofluidics. Appl. Phys. Lett. 2002, 81, 3058.

(20) Goldberger, J.; Fan, R.; Yang, P. Inorganic Nanotubes : A Novel Platform for Nanofluidics Rational Synthesis of Inorganic Nanotubes. Acc. Chem. Res. 2006, 39, 239248 .

(21) Li, Q.; Westlake, K. R.; Crawford, M. H.; Lee, S. R.; Koleske, D. D.; Figiel, J. J.; Cross, K. C.; Fathololoumi, S.; Mi, Z.; Wang, G. T. Optical Performance of Top-down Fabricated InGaN/GaN Nanorod Light Emitting Diode Arrays. Opt. Express 2011, 19, $25528-25534$.

(22) Wierer, J. J.; Li, Q.; Koleske, D. D.; Lee, S. R.; Wang, G. T. III-Nitride Core-shell Nanowire Arrayed Solar Cells. Nanotechnology 2012, 23, 194007.

(23) Goldberger, J.; He, R.; Zhang, Y.; Lee, S.; Yan, H.; Choi, H.-J.; Yang, P. Single-Crystal Gallium Nitride Nanotubes. Nature 2003, 422, 599-602.

(24) Kouno, T.; Kishino, K.; Suzuki, T.; Sakai, M. Lasing Actions in GaN Tiny Hexagonal Nanoring Resonators. IEEE Photonics J. 2010, 2, 1027-1033.

(25) Kuga, T.; Torii, Y.; Shiokawa, N.; Hirano, T.; Shimizu, Y.; Sasada, H. Novel Optical Trap of Atoms with a Doughnut Beam. Phys. Rev. Lett. 1997, 78, 4713-4716.

(26) Hell, S. W.; Wichmann, J. Breaking the Diffraction Resolution Limit by Stimulated Emission: Stimulated-Emission-Depletion Fluorescence Microscopy. Opt. Lett. 1994, 19, 780. 